\title{
Can magnetic resonance imaging predict preoperative consistency and vascularity of intracranial meningioma?
}

\author{
Ahmed A. ElBeheiry', Ahmed A. Fayed ${ }^{2}$, Ahmed H. Alkassas ${ }^{1}$ and Doaa M. Emara ${ }^{1 *}$ (1)
}

\begin{abstract}
Background: Meningiomas are considered the most common primary intracranial neoplasms. The surgical resection is the main curative therapy. Evaluation of meningioma consistency and vascularity is important before surgery to be aware about the difficulties that neurosurgeon will face during resection, the possibility of total resection and to determine which equipment will be suitable for surgery. The purpose of this study was to identify the relationship between the MRI predictors of meningioma consistency [utilizing tumor/cerebellar peduncle T2-weighted imaging intensity (TCTI) ratios] as well as tumor vascularity (utilizing arterial spin labeling perfusion) in correlation with intraoperative findings. The study was carried out on 40 patients with MRI features of intracranial meningiomas. Non-contrast conventional MRI followed by arterial spin labeling MR perfusion and post contrast sequences were done for all cases. Final diagnosis of the cases was established by histopathological data while consistency and vascularity was confirmed by operative findings.
\end{abstract}

Results: According to surgical data, the studied cases of intracranial meningiomas were classified according to tumor consistency into 19 cases (47.5\%) showing soft consistency, 14 cases (35\%) showing intermediate consistency and 7 cases (17.5\%) showing firm/hard consistency. TCTI ratio was the most significant MRI parameter in correlation with operative consistency of meningiomas, with soft lesions showing TCTI ranging from 1.75 to 2.87 , intermediate consistency lesions TCTI ranging from 1.3 to 1.6, and firm lesions TCTI ranging from 0.9 to 1.2. According to intraoperative vascularity, cases were classified into 27 cases (67.5\%) showing hypervascularity, 6 cases (15\%) showing intermediate vascularity and 7 cases (17.5\%) showing hypovascularity. Arterial spin labeling (ASL) was the most significant MRI parameter in correlation with operative vascularity of meningiomas, with hypervascular lesions showing normalized cerebral blood flow (n-CBF) ranging from 2.10 to 14.20, intermediately vascular lesions ranging from 1.50 to 1.60, and hypovascular lesions ranging from 0.70 to 0.90 .

Conclusions: TCTI ratio showed good correlation with intraoperative meningioma consistency. ASL MR perfusion as a noninvasive technique is a reliable method to predict vascularity of meningioma in cases where IV contrast is contraindicated.

Keywords: Meningiomas, Tumor/cerebellar peduncle T2-weighted imaging intensity (TCTI) ratio, Arterial Spin Labeling

\footnotetext{
*Correspondence: dr.emara_doaa@yahoo.com; dr.emara-doaa@hotmail.com ${ }^{1}$ Department of Radiodiagnosis, Faculty of Medicine, University of Alexandria, Alexandria, Egypt

Full list of author information is available at the end of the article
}

\section{Background}

Meningiomas are considered the most common primary intracranial neoplasms, with an incidence of $13 \%-26 \%$. Generally, meningiomas are slowly growing lesions, with its clinical course depending on the location $[1,2]$. 
MRI is the imaging modality of choice for assessment of intracranial lesions suspected to be meningiomas or in cases with highly suspected pathology [3]. Conventional MRI using the routine sequences, T1-weighted and T2-weighted imaging sequences, as well as post contrast T1-weighted sequences, is enough for diagnosis of meningiomas [3].

Therapy for patients with meningiomas will differ from one patient to the other due to the nature of the tumor. The potential consequences of different treatments are greatly variable for different patients, however surgery is still the primary therapeutic method. Recently less invasive techniques are used instead of conventional open approaches as an alternative for surgical resection of intracranial meningiomas, such as keyhole or transnasal endoscopic approaches. Such techniques have been commonly used in skull base meningiomas, yet multiple factors should be taken in consideration in order to select which technique that will be used in each patient such as site of the tumor, its aggressiveness, vascularity and relation to vital structures $[4,5]$.

Prior to surgery, it is important to assess the consistency, firmness, or texture of the meningioma as it is of value to decide the surgical plane as well as expectation to extension of tumor resection. Also, it helps in detection of potential risks and the expected length of operation time. This is true for tumors which express extremes of consistency (i.e., extremely soft vs. extremely firm) [6, 7].

The main blood supply of intracranial meningiomas is from dural branches of the external carotid artery (ECA). In the meningioma subtypes, angiomatous meningioma is defined as having more than $50 \%$ tumor volume occupied by blood vessels. Angiomatous meningiomas usually do not have aggressive behavior, however surgical resections are sometimes difficult in certain cases due to bleeding intra-operatively, mainly if their blood supply is from pial-cortical arteries [8].

In the last decade, there has been increased interest in a noninvasive MRI perfusion technique which is arterial spin labeling (ASL) that provides data about absolute quantification of tissue perfusion without administration of IV contrast, unlike dynamic susceptibility contrastenhanced perfusion weighted imaging (PWI), so it can be safely used in patients with impaired renal function, and in whom there is higher risk of developing nephrogenic systemic fibrosis [9].

The aim of this study was to identify the relationship between the MRI predictors of intracranial meningioma consistency (utilizing tumor/cerebellar peduncle T2-weighted imaging intensity (TCTI) ratios) as well as tumor vascularity (utilizing arterial spin labeling perfusion) in correlation with intraoperative findings.

\section{Methods}

Approval for this cross-sectional prospective study was obtained from the local human research ethics committee. All procedures were accomplished according to the Declaration of Helsinki regarding research involving human subjects [10]. All patients agreed to give written informed consent.

\section{Study population}

This prospective study was conducted during the period between January 2020 and October 2020 on a total of 40 patients presenting by imaging findings suggestive of underlying meningioma referred from Neurosurgery department and clinic in our institute for further preoperative consistency and vascularity assessment by MRI. Only patients who underwent total/gross total tumor resection with subsequent histologic findings confirming the diagnosis of meningioma were involved.

\section{MRI imaging protocol}

MRI examinations were done on 3T MRI machine (Discovery MR750 w 3.0T, General Electric, Milwaukee, USA). MR images were acquired with the subsequent protocols.

\section{Pre-contrast series}

Imaging parameters included axial T1-weighted spin echo (repetition time $(\mathrm{TR})=600 \mathrm{~ms}$, echo time $(\mathrm{TE})=15 \mathrm{~ms}$ ), axial, sagittal and coronal $\mathrm{T} 2$-weighted turbo spin echo $(\mathrm{TR}=4000 \mathrm{~ms}, \mathrm{TE}=100 \mathrm{~ms})$, axial FLAIR $(\mathrm{TR}=11,000 \mathrm{~ms}, \mathrm{TE}=140 \mathrm{~ms}$, inversion time $(\mathrm{TI})=2200 \mathrm{~ms}$ ), Diffusion weighted imaging (DWI) with diffusion gradient $b$ values of 0 and $1000 \mathrm{~s} / \mathrm{mm}^{2}$, along three orthogonal axes ( $x, y$ and $z$ directions) over $(\mathrm{TR}=5072$; number of sections $=16-22)$ and Susceptibility Weighted Images; SWI (TR $38.5 \mathrm{ms,}$ TE $22.9 \mathrm{~ms}, 320 \times 224$ acquisition matrix, field of view $240 \times 192 \mathrm{~mm}$, slice thickness $3 \mathrm{~mm}$ and a gap of $0.2 \mathrm{~mm}$ ).

\section{Arterial spin labeling MR perfusion}

The imaging parameters were: $\mathrm{TR} / \mathrm{TE}=4894 / 10.7 \mathrm{~ms}$, inversion time (TI) was $2025.0 \mathrm{~ms}$, flip angle $=40$ 。, FOV $=250 \times 250 \mathrm{~cm}$, matrix $=512 \times 8$, slice thickness $/$ gap $=4 / 0.0 \mathrm{~mm}$, number of dynamics $=30$. The total sequence acquisition time was $4 \mathrm{~min}$ with the labeling slab positioned at the level of upper cervical region.

\section{Post-contrast series}

After injection of Gadolinium diethylene triamine pentacetic acid (GAD-DTPA) in a dose of $0.1 \mathrm{mmol} /$ 
$\mathrm{kg}$ body weight, images were obtained in axial, coronal and sagittal T1-weighted spin echo sequences.

\section{Data analysis and Image evaluation}

MRI data were reviewed for each patient. The following steps of imaging evaluation were followed:

\section{Assessment of meningioma consistency by:}

(a) Visual assessment of the meningioma signal

- Visual determination of meningioma signal by comparing the signal of the lesion in relation to the signal of the cortex in $\mathrm{T} 2$ sequence.

(b) Quantitative assessment of the meningioma signal

- Assessment of the tumor diffusion by measurement of the apparent diffusion coefficient (ADC) in DWI.

- Quantitative assessment of the tumor signal utilizing tumor/cerebellar peduncle T2-weighted imaging intensity (TCTI) ratios. Using T2 sequence on MRI, tumor to cerebellar peduncle TCTI ratios were measured. Regions of interest (ROIs) were drawn inside the tumor to obtain an average intensity. For intensity comparison, the middle cerebellar peduncle was chosen, and ROIs were again measured. Such quantitative assessment was based on the previous study of Smith KA et al. [11], as shown in Fig. 1.

\section{Assessment of meningioma vascularity by:} Quantitative Analysis of cerebral blood flow (CBF) on ASL MR perfusion: On GE workstation "Advantage 4.7", ROIs covering all of the contrast-enhancing areas, apart from areas of necrosis or cysts, were drawn in the most representative sections of each $\mathrm{CBF}$ map to define the absolute $\mathrm{CBF}$ of the tumor ( $\mathrm{CBF}$ tumor). At the same time, to accommodate for the inter-individual variation among MR scanners and basal CBF, supplementary ROIs were drawn within the white matter of normal appearing contralateral parenchyma at the same section levels for CBF normalization. Thereafter, normalized CBF $(\mathrm{n}-\mathrm{CBF})$ were measured as $\mathrm{n}-\mathrm{CBF}=\mathrm{CBF}$ tumor $/ \mathrm{CBF}$ white matter.

\section{Intra-operative assessment}

During surgery, the surgeon used the intraoperative setting to objectively quantify the consistency and vascularity of meningiomas during resection. Consistency grades were assigned based on the use of surgical instruments and suction, the most important of which was cavitron ultrasonic surgical aspirator (CUSA). A grading scale of consistency was followed based on CUSA intensity. Grade A, soft consistency meningiomas with cutoff values $<40$; grade $B$ intermediate consistency lesions with values 40 to 70 , and $>70$ for grade $C$, firm consistency

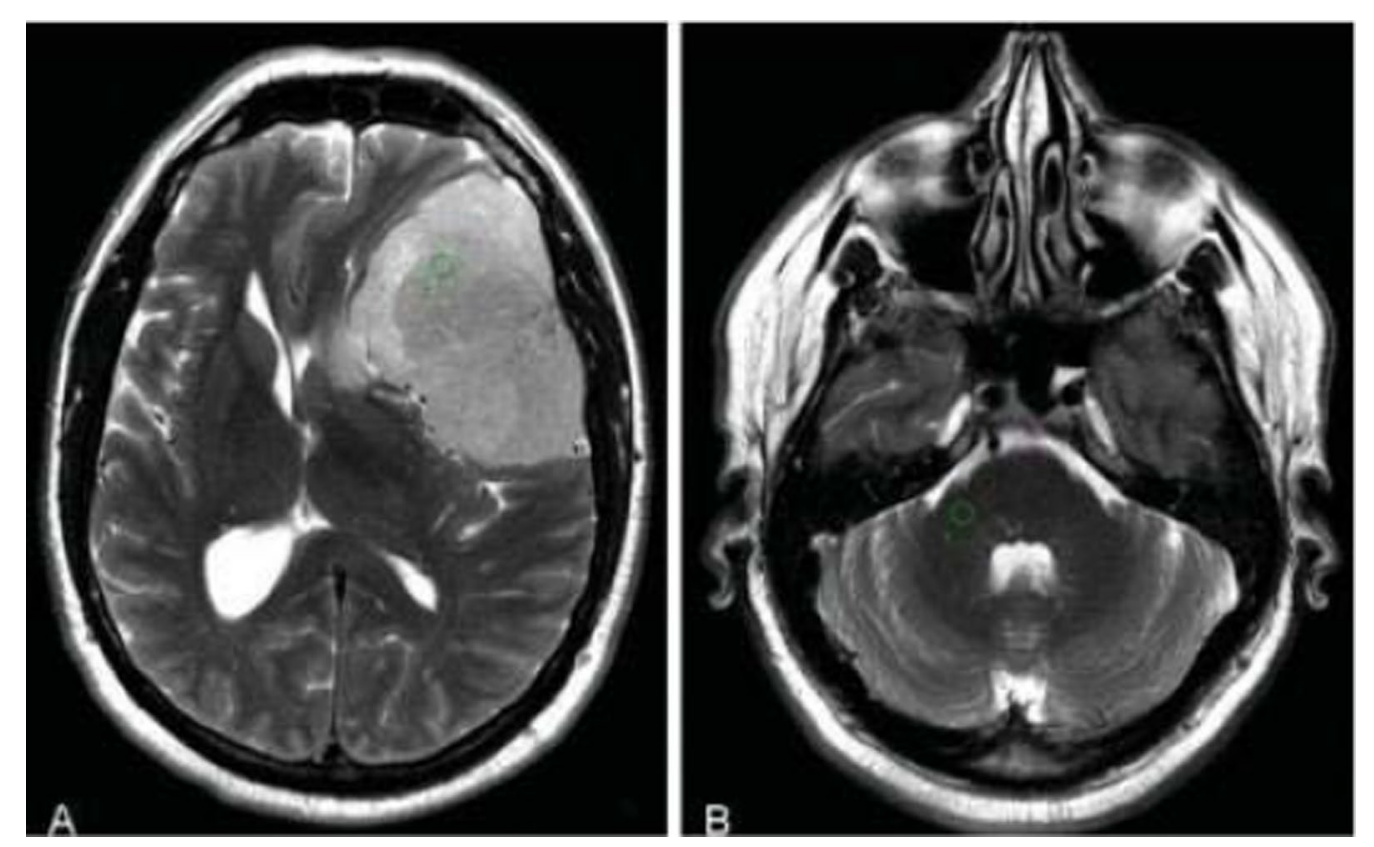

Fig. 1 How to use the tumor to cerebellar peduncle T2-weighted imaging intensity (TCTI) ratio. A Region of interest (ROI) in tumor on T2 sequence. B ROI in middle cerebellar peduncle on T2 sequence. Calculating the TCTI ratio. TCTI ratio intensity value for ROI within tumor (A)/Intensity value for ROI within middle cerebellar peduncle (B) [1 1] 
tumors. These amplitudes denote the lowest CUSA setting possible to adequately remove the tumor. Operative assessment of tumor vascularity depended on degree of intraoperative blood loss as well as tumoral vessels detection.

\section{Clinico-radiological correlation}

The correlation between the different MRI-based parameters of meningioma consistency and vascularity in relation to intraoperative findings was studied.

\section{Statistical analysis}

Data were given to the computer and analyzed utilizing IBM SPSS software version 20.0. (Armonk, NY: IBM Corp). The Kolmogorov- Smirnov, Shapiro and D'agstino tests were utilized to confirm the normality of distribution of variables. Comparisons between groups for categorical variables were surveyed using Chi-square test (Fisher or Monte Carlo). Receiver operating characteristic curve (ROC) was utilized to assess the diagnostic performance of the markers. Area above $50 \%$ gives acceptable performance and area about $100 \%$ is the best performance for the test. Significance of the obtained results was judged at the 5\% level. Mann-Whitney test was utilized to compare between two groups for abnormally distributed quantitative variables.

\section{Results}

\section{Demographic data}

The current study included 40 adult's meningioma patients: 28 females and 12 males (ranging in age from 40 to 70 years with mean age 53 years). Ten patients presented with headache, 10 had motor weakness, 5 had ipsilateral optic atrophy and bi-temporal hemianopia, 5 had anosmia and optic atrophy, 5 complained from decreased vision, diplopia and facial numbness and 5 patients had convulsions.

\section{Operative data}

\section{Meningioma consistency}

According to surgical data, the studied cases of intracranial meningiomas were classified according to tumor consistency into the following: 19 cases (47.5\%) showed intraoperative soft consistency, 14 cases (35\%) showed intermediate consistency and 7 cases (17.5\%) showed firm/hard consistency.

\section{Meningioma vascularity}

According to tumor intraoperative vascularity, cases were classified into the following: 27 cases (67.5\%) showed intraoperative hypervascularity, 6 cases (15\%) showed intraoperative intermediate vascularity and 7 cases (17.5\%) showed intraoperative hypovascularity.

\section{Imaging results} Meningioma consistency

Soft meningiomas Nineteen cases with surgically proven soft meningiomas showed the following MRI features:

- T2 signal characteristics

Fourteen cases showed T2 hyperintense signal, five cases showed iso-intense signal while no cases were hypointense on T2WI.

- DWI and ADC characteristics

Fifteen cases showed diffusion restriction with ADC ranging from $(0.6-0.9) \times 10^{-5} \mathrm{~cm}^{2} / \mathrm{s}$, two cases showed iso-diffusion with ADC of $1.1 \times 10^{-5} \mathrm{~cm}^{2} / \mathrm{s}$ while two cases showed free diffusion with $\mathrm{ADC}$ averaging $1.3 \times 10^{-5} \mathrm{~cm}^{2} / \mathrm{s}$.

- Tumor to cerebellar peduncle T2 intensity (TCTI) ratio characteristics

Soft meningioma cases showed TCTI ratios ranging from 1.75 to 2.87 (Fig. 2).

Intermediate consistency meningiomas Fourteen cases with surgically proven intermediate consistency meningiomas showed the following MRI features:

- T2 signal characteristics

Eight cases showed T2 iso intense signal, five cases showed hyperintense signal and one case showed hypointensity on T2WI.

- DWI and ADC characteristics

Ten cases showed diffusion restriction with ADC ranging from $(0.7-0.9) \times 10^{-5} \mathrm{~cm}^{2} / \mathrm{s}$, and four cases showed iso diffusion with ADC ranging from (1.0$1.2) \times 10^{-5} \mathrm{~cm}^{2} / \mathrm{s}$.

- Tumor to cerebellar peduncle T2 intensity (TCTI) ratio characteristics

Intermediate consistency meningioma cases showed TCTI ratios ranging from 1.31 to 1.60 (Fig. 3).

Hard/firm meningiomas Seven cases with surgically proven hard meningiomas showed the following MRI features:

- T2 signal characteristics

Five cases showed T2 iso intense signal, and two cases showed hypo intense signal.

- DWI and ADC characteristics

Four cases showed iso diffusion with ADC ranging from $(0.9-1.1) \times 10^{-5} \mathrm{~cm}^{2} / \mathrm{s}$ and three cases showed free diffusion with ADC ranging from (1.31.4) $\times 10^{-5} \mathrm{~cm}^{2} / \mathrm{s}$.

- Tumor to cerebellar peduncle T2 intensity (TCTI) ratio characteristics 


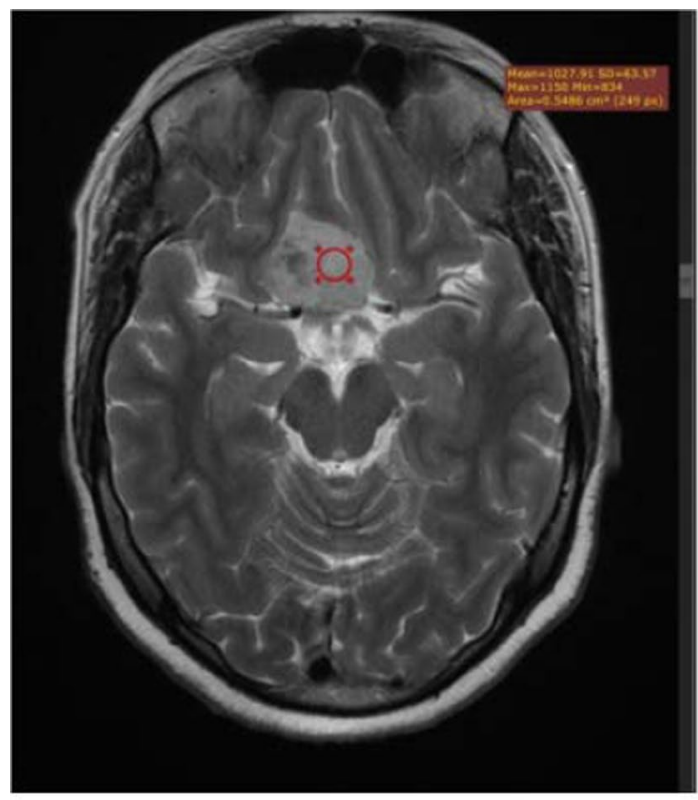

(A)

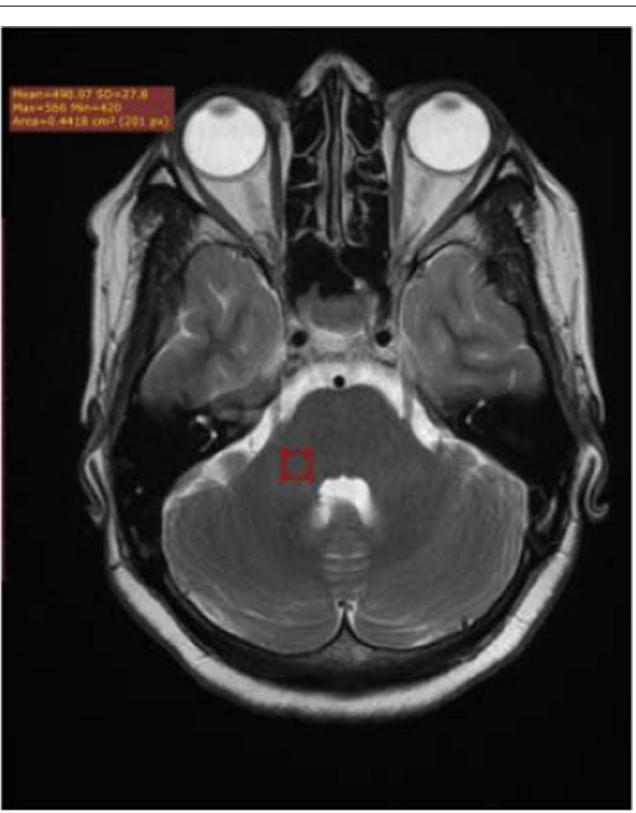

(B)

Fig. 2 MRI of soft Tuberculum sellae meningioma. A, B Axial T2-weighted MR images at the level of Tuberculum sellae meningioma and at the level of middle cerebellar peduncles showing TCTI ratio of 2.2, proved surgically to have soft consistency

Hard meningioma cases showed TCTI ratios ranging from 0.9 to 1.2 (Fig. 4).

Statistical correlation between MRI parameters and meningioma intraoperative consistency Significant difference was noted between the three types of meningioma consistency and different MRI parameters including T2 signal, diffusion, and ADC as well as tumor to cerebellar peduncle T2 intensity (TCTI) as follows:

- Significant difference $(P=0.001)$ was noted between soft and firm meningiomas regarding T2 signal with $73.7 \%$ of soft lesions having T2 hyper intensity while firm lesions showed $71.4 \%$ having isointensity. Also noted significant difference $(P=0.047)$ between soft and intermediate meningiomas regarding $\mathrm{T} 2$ signal with $57.1 \%$ of cases with intermediate consistency lesions having T2 isointensity. No significant difference was noted between intermediate and firm consistency lesions regarding T2 signal.

- Significant difference $(P=0.001)$ was also noted between soft and intermediate lesions on a side and firm meningiomas on the other side regarding diffusion and ADC with $78.9 \%$ of soft lesions and $71.4 \%$ of intermediate consistency lesions showing restricted diffusion with average ADC of $0.89 \times 10^{-5} \mathrm{~cm}^{2} / \mathrm{s}$ and $0.88 \times 10^{-5} \mathrm{~cm}^{2} / \mathrm{s}$ respectively while $57.1 \%$ of firm consistency lesions showed iso diffusion with ADC averaging $1.1 \times 10^{-5} \mathrm{~cm}^{2} / \mathrm{s}$. No significant difference was noted between soft and intermediate consistency lesions.

- TCTI ratio was the most significant MRI parameter in correlation with operative consistency of meningiomas, with soft lesions showing TCTI ranging from 1.75 to 2.87 , intermediate consistency lesions TCTI ranging from 1.3 to 1.6 , and firm lesions TCTI ranging from 0.9 to 1.2 . ROC analysis showed $100 \%$ sensitivity, specificity, PPV and NPV for TCTI ratio $>1.6$ in prediction of soft consistency, TCTI ratio $\leq 1.2$ for firm consistency with intermediate consistency lesions having TCTI between $>1.2$ and $\leq 1.6$ (Table 1 and Fig. 5).

\section{Meningioma vascularity}

Hypervascular meningiomas Twenty seven cases with surgically proven hypervascular meningiomas showed the following MRI features:

\section{- T2 signal characteristics}

Eighteen cases showed T2 hyperintense signal, nine cases showed iso intense signal while no cases were hypo intense on T2WI.

- DWI and ADC characteristics

Twenty cases showed diffusion restriction with $\mathrm{ADC}$ ranging from $(0.6-0.9) \times 10^{-5} \mathrm{~cm}^{2} / \mathrm{s}$, 


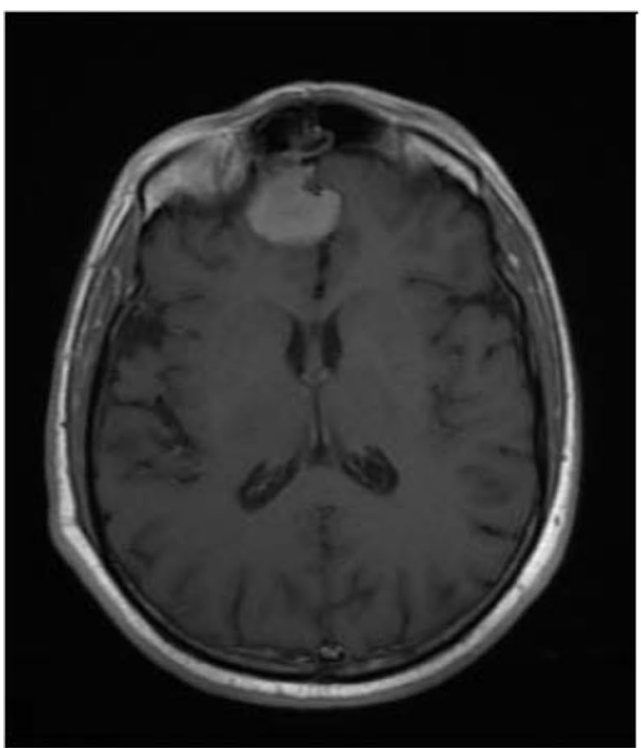

(A)

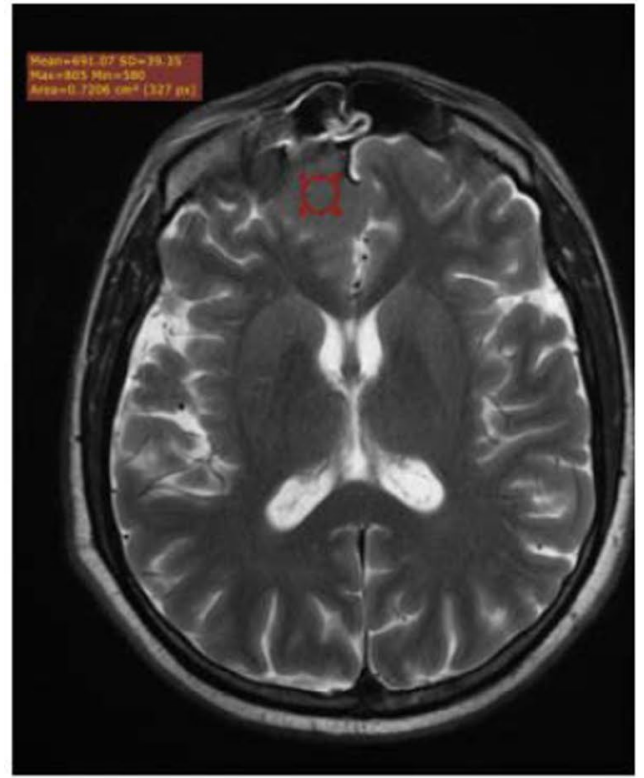

(C)

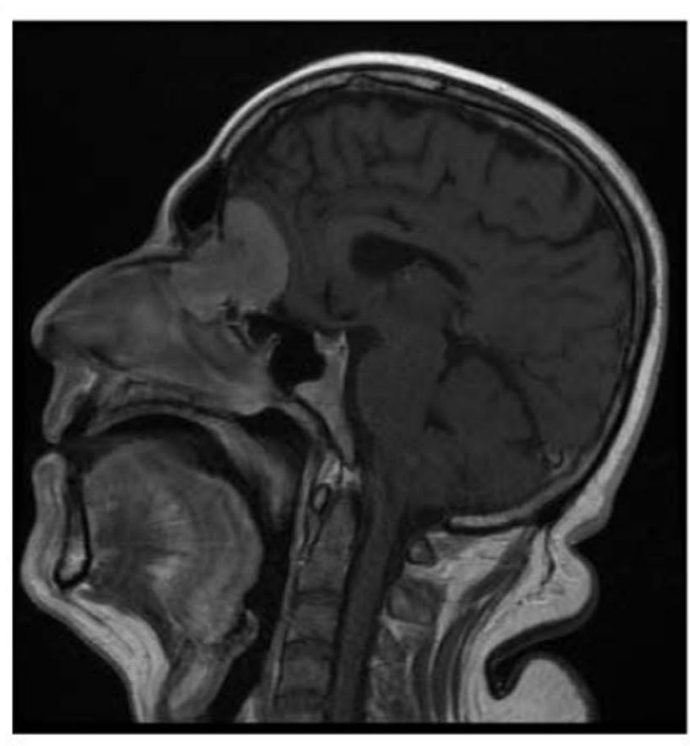

(B)

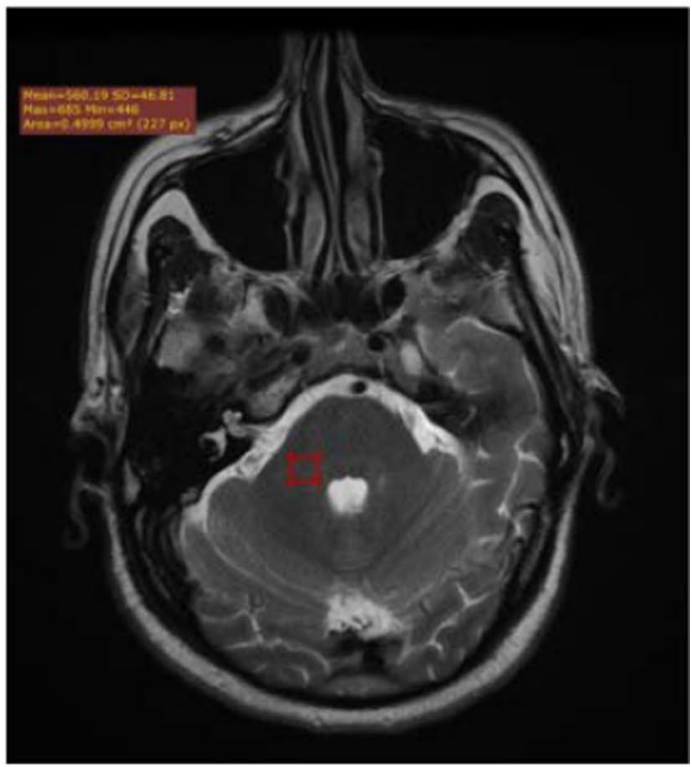

(D)

Fig. $3 \mathrm{MRl}$ of intermediate consistency olfactory groove meningioma. A, B Axial and sagittal T1 post contrast showing intensely enhancing olfactory groove meningioma. C, D Axial T2-weighted MR images at the same level and at the level of middle cerebellar peduncles with ROI on the lesion and right middle cerebellar peduncle showing TCTI ratio of 1.4, proved surgically to have intermediate consistency

five cases showed iso diffusion with ADC of $1.1 \times 10^{-5} \mathrm{~cm}^{2} / \mathrm{s}$ and two cases showed free diffusion with ADC averaging $1.3 \times 10^{-5} \mathrm{~cm}^{2} / \mathrm{s}$.

- Arterial spin labeling and normalized cerebral blood flow (nCBF) characteristics

All 27 cases (100\%) showed hyperperfusion with nCBF ranging from 2.10 to 14.20 (Fig. 6).
Intermediately vascular meningiomas Six cases with surgically proven intermediately vascular meningiomas showed the following MRI features:

\section{- T2 signal characteristics}




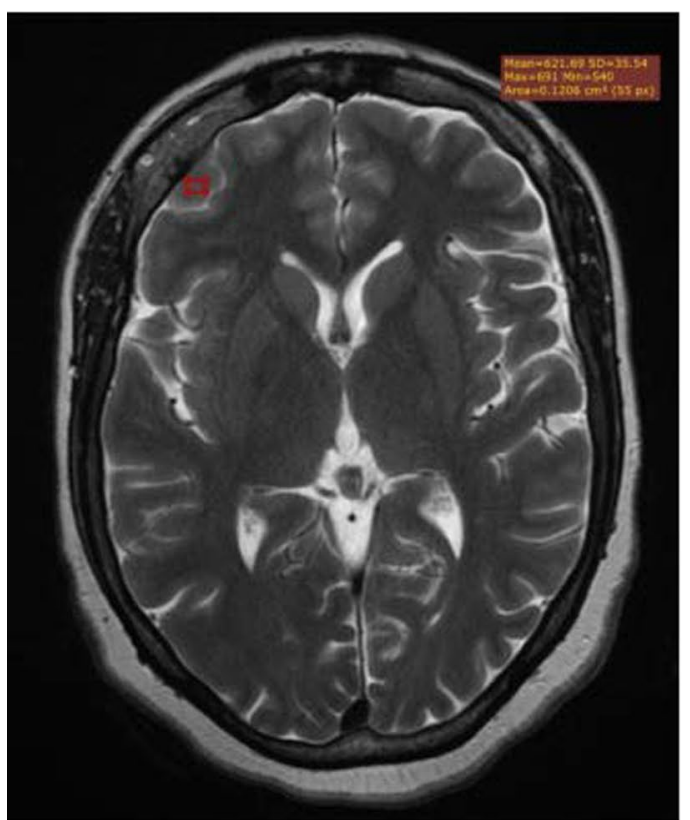

(A)

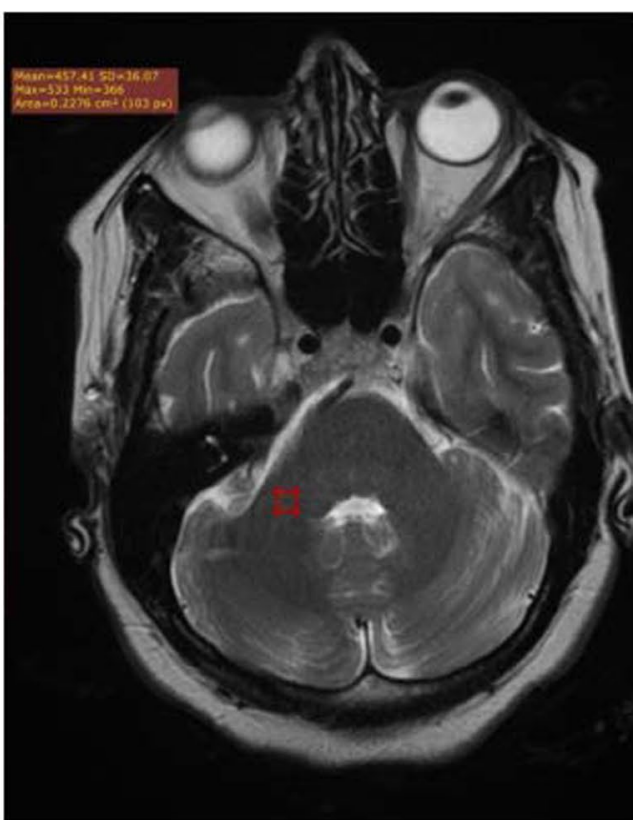

(B)

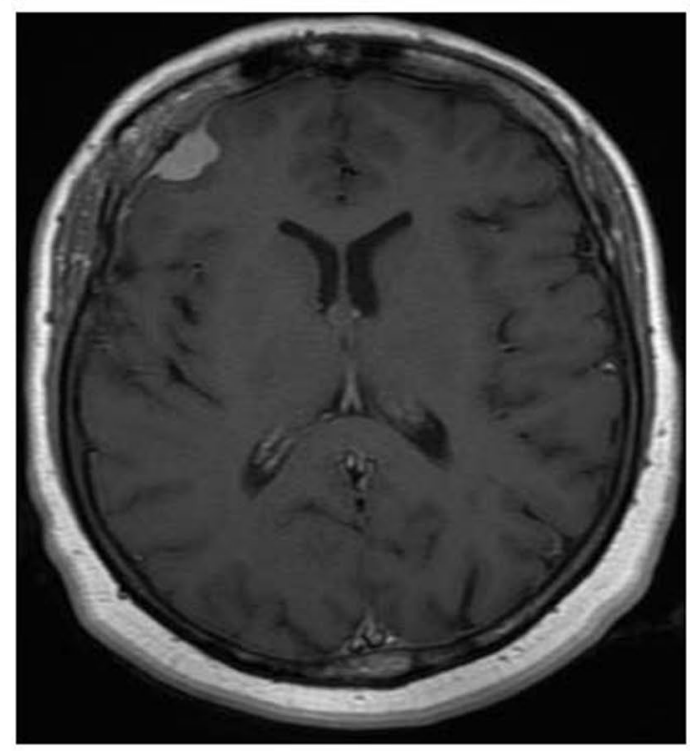

(C)

Fig. $4 \mathrm{MRl}$ of firm consistency right frontal convexity meningioma. A, B Axial T2-weighted MR images at the level of right frontal convexity meningioma and at the level of middle cerebellar peduncles showing TCTI ratio of 1.2, proved surgically to have firm consistency. C Axial T1 post contrast showing intense enhancement

One case showed hyperintense signal, four case showed T2 iso intense signal, and one case showed hypo intensity on T2WI.

- DWI and ADC characteristics
Two cases showed diffusion restriction with ADC ranging from $(0.7-0.9) \times 10^{-5} \mathrm{~cm}^{2} / \mathrm{s}$ and four cases showed iso diffusion with ADC ranging from (1.01.2) $\times 10^{-} 5 \mathrm{~cm}^{2} / \mathrm{s}$. 
Table 1 Relation between meningioma operative consistency and different MRI parameters

\begin{tabular}{|c|c|c|c|c|c|c|c|}
\hline \multirow[t]{3}{*}{ MRI parameters } & \multicolumn{6}{|c|}{ Meningioma operative consistency } & \multirow[t]{3}{*}{$p$} \\
\hline & \multicolumn{2}{|l|}{$\begin{array}{l}\text { Soft } \\
(n=19)\end{array}$} & \multicolumn{2}{|l|}{$\begin{array}{l}\text { Intermediate } \\
(n=14)\end{array}$} & \multicolumn{2}{|l|}{$\begin{array}{l}\text { Firm } \\
(n=7)\end{array}$} & \\
\hline & No & $\%$ & No & $\%$ & No & $\%$ & \\
\hline \multicolumn{8}{|l|}{ T2 signal } \\
\hline Hyperintense & 14 & 73.7 & 5 & 35.7 & 0 & 0.0 & $p=0.001^{*}$ \\
\hline Isointense & 5 & 26.3 & 8 & 57.1 & 5 & 71.4 & \\
\hline Hypointense & 0 & 0.0 & 1 & 7.1 & 2 & 28.6 & \\
\hline Significance between groups & \multicolumn{6}{|c|}{$p_{1}=0.047^{*}, p_{2}=0.001^{*}, p_{3}=0.117$} & \\
\hline \multicolumn{8}{|l|}{ Diffusion } \\
\hline Free diffusion & 2 & 10.5 & 0 & 0.0 & 3 & 42.9 & $p<0.001^{*}$ \\
\hline Iso diffusion & 2 & 10.5 & 4 & 28.6 & 4 & 57.1 & \\
\hline Restricted & 15 & 78.9 & 10 & 71.4 & 0 & 0.0 & \\
\hline Significance between groups & \multicolumn{7}{|c|}{$p_{1}=0.322, p_{2}=0.001^{*}, p_{3}=0.001^{*}$} \\
\hline$A D C$ & \multicolumn{2}{|l|}{$\times 10^{-5} \mathrm{~cm}^{2} / \mathrm{s}$} & \multicolumn{2}{|l|}{$\times 10^{-5} \mathrm{~cm}^{2} / \mathrm{s}$} & \multicolumn{2}{|l|}{$\times 10^{-5} \mathrm{~cm}^{2} / \mathrm{s}$} & \\
\hline Min.-Max & \multicolumn{2}{|l|}{$0.60-1.30$} & \multicolumn{2}{|l|}{$0.70-1.20$} & \multicolumn{2}{|l|}{$0.90-1.40$} & $p=0.024^{*}$ \\
\hline Mean $\pm S D$ & \multicolumn{2}{|l|}{$0.89 \pm 0.19$} & \multicolumn{2}{|l|}{$0.88 \pm 0.22$} & \multicolumn{2}{|l|}{$1.13 \pm 0.21$} & \\
\hline Median & \multicolumn{2}{|l|}{0.85} & \multicolumn{2}{|l|}{0.80} & \multicolumn{2}{|l|}{1.10} & \\
\hline Significance between groups & \multicolumn{6}{|c|}{$p_{1}=0.987, p_{2}=0.031^{*}, p_{3}=0.031^{*}$} & \\
\hline \multicolumn{8}{|l|}{ TCTI ratio } \\
\hline Min.-Max & \multicolumn{2}{|l|}{$1.75-2.87$} & \multicolumn{2}{|l|}{$1.31-1.60$} & \multicolumn{2}{|l|}{$0.90-1.20$} & $p<0.001^{*}$ \\
\hline Mean $\pm S D$ & \multicolumn{2}{|l|}{$1.99 \pm 0.29$} & \multicolumn{2}{|l|}{$1.50 \pm 0.10$} & \multicolumn{2}{|l|}{$1.09 \pm 0.11$} & \\
\hline Median & 1.90 & & 1.53 & & \multirow{2}{*}{\multicolumn{2}{|c|}{1.10}} & \\
\hline Significance between groups & \multicolumn{4}{|c|}{$p_{1}<0.001^{*}, p_{2}<0.001^{*}, p_{3}=0.001^{*}$} & & & \\
\hline
\end{tabular}

ADC, apparent diffusion coefficient; $p, p$ value for association between different categories; $\mathrm{n}$, number of patients; $p_{1}, p$ value for association between soft and intermediate; $p_{2}, p$ value for association between soft and firm; $p_{3}, p$ value for association between intermediate and firm; SD, standard deviation; TCTI, Tumor/ cerebellar peduncle T2-weighted imaging intensity

*Statistically significant at $p \leq 0.05$
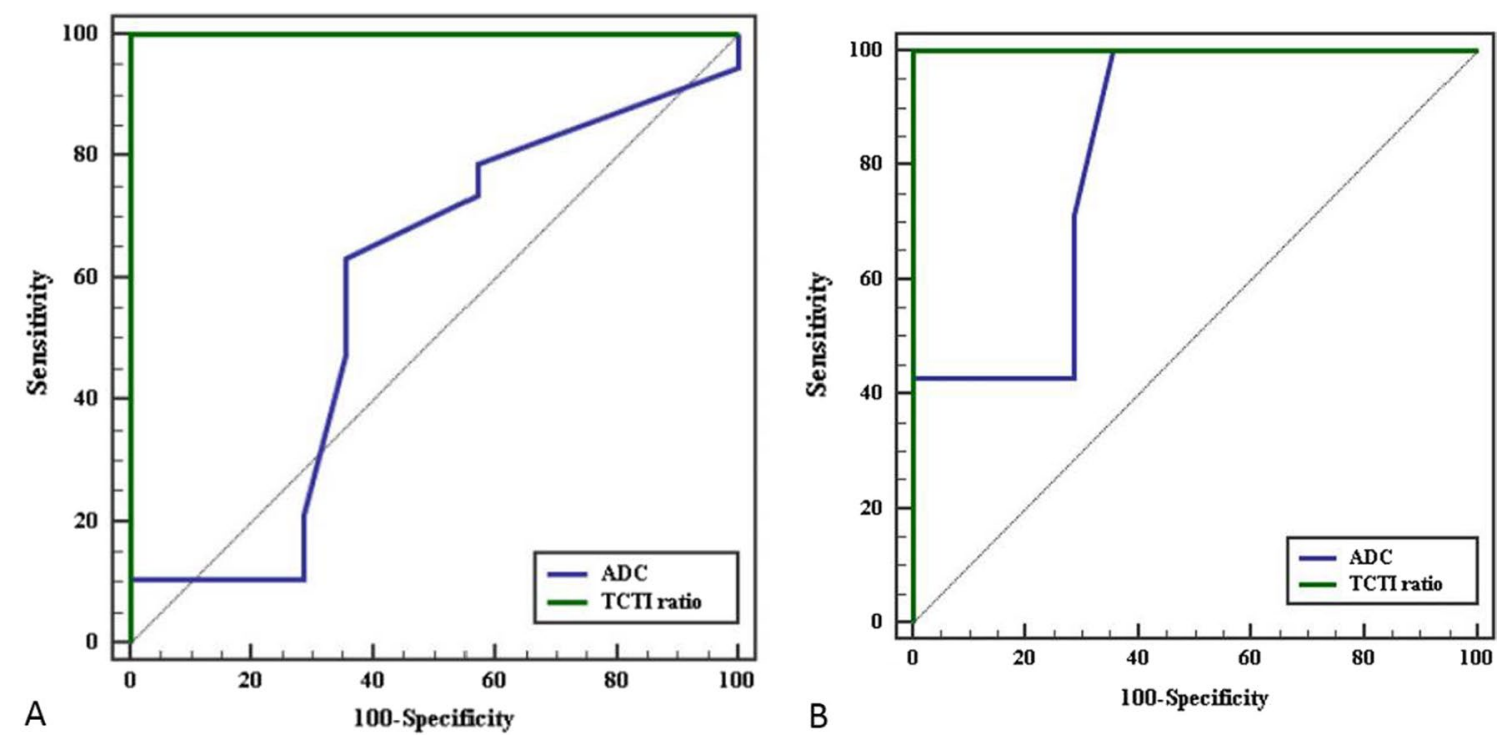

Fig. 5 ROC curve for different parameters (ADC and TCTI) to predict meningioma consistency. A ROC curve for ADC $<0.8$ and TCTI $>1.6$ for prediction of soft meningioma. B ROC curve for $\mathrm{ADC}>0.9$ and $\mathrm{TC} \mathrm{TI} \leq 1.2$ for prediction of firm meningioma 


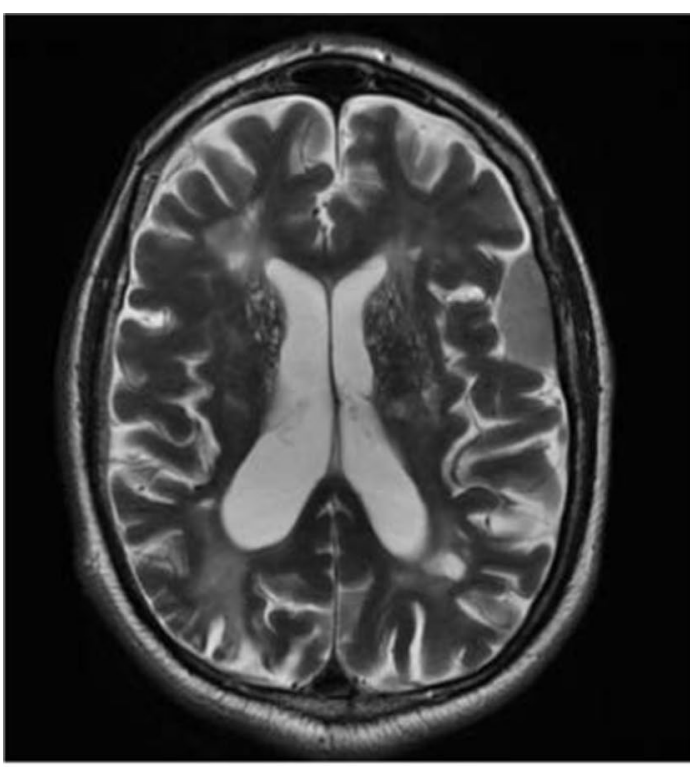

(A)

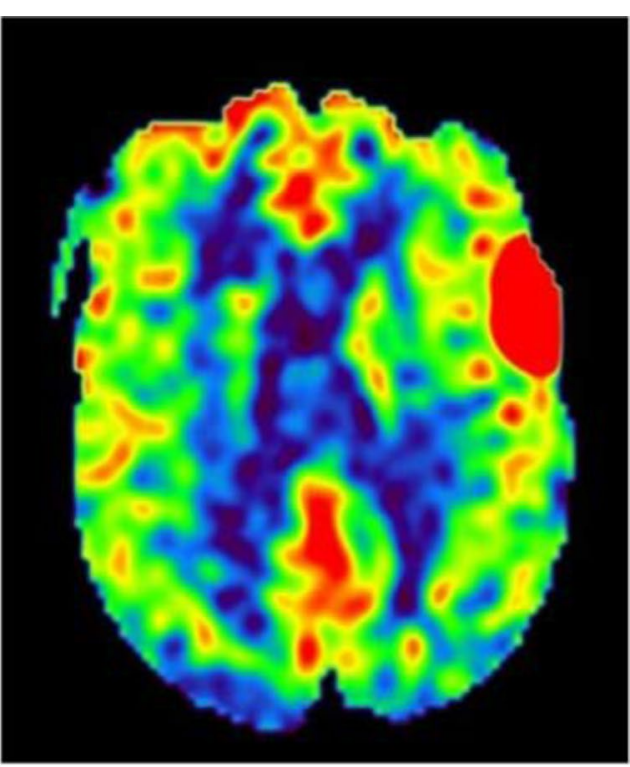

(B)

Fig. 6 MRI of hypervascular meningioma: A Axial T2-weighted MRI showing left frontal convexity meningioma. B Color coded ASL perfusion showing hyperperfusion of the lesion with $\mathrm{nCBF}$ of 7.4 , proved surgically to have high vascularity

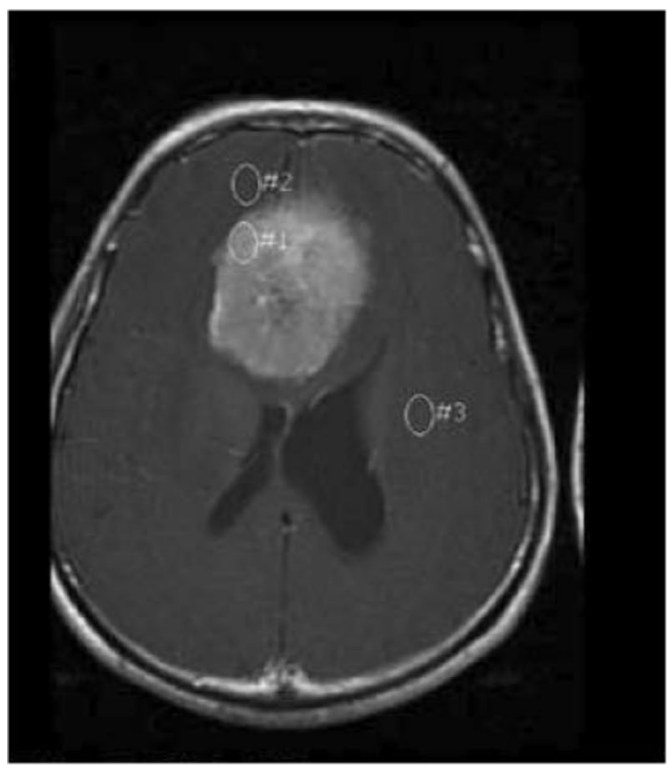

(A)

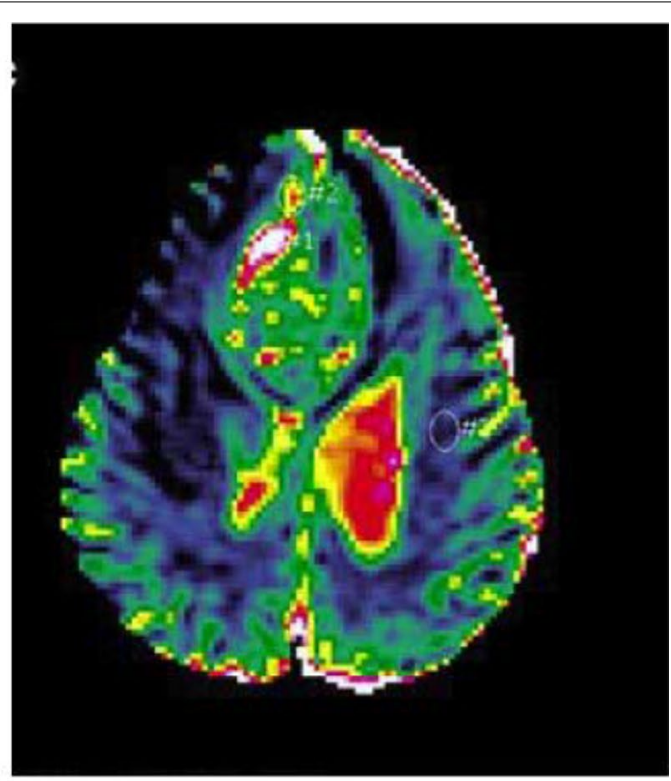

(B)

Fig. $7 \mathrm{MRI}$ of intermediately vascular meningioma: A Axial T1 post contrast MRI showing intensely enhancing falcine meningioma. B ASL perfusion showing iso perfusion of the lesion with nCBF of $1.6 \mathrm{ml} / 100 \mathrm{gm} / \mathrm{min}$, proved surgically to have intermediate vascularity 
- Arterial spin labeling and nCBF characteristics All 6 cases showed iso-perfusion with nCBF ranging from 1.5 to 1.6 (Fig. 7 ).

Hypovascular meningiomas Seven cases with surgically proven hypovascular meningiomas showed the following MRI features:

\section{- T2 signal characteristics}

Two cases showed hyperintense signal, two cases showed T2 iso intense signal, and three cases showed hypointensity on T2WI.

- DWI and ADC characteristics

Five cases showed diffusion restriction with ADC ranging from $(0.7-0.9) \times 10^{-5} \mathrm{~cm}^{2} / \mathrm{s}$ and two cases showed iso diffusion with ADC ranging from (1.01.2) $\times 10^{-5} \mathrm{~cm}^{2} / \mathrm{s}$.

- Arterial spin labeling and nCBF characteristics All 7 cases showed hypo perfusion with nCBF ranging from 0.7 to 0.9 (Fig. 8).

Statistical correlation between MRI parameters and meningioma intraoperative vascularity Significant correlation was noted between meningioma vascularity and different MRI parameters as follows:
- Significant difference was noted between hyper vascular and intermediately vascular $(P=0.001)$ as well as hypo vascular meningiomas $(P=0.001)$ regarding T2 signal with $66.7 \%$ of cases of hyper vascular lesions having T2 hyper intensity and $66.7 \%$ of cases of intermediate vascularity lesions having iso intensity while $42.9 \%$ of hypo vascular meningiomas were T2 hypo intense.

- No significant correlation was found between meningioma vascularity and diffusion or ADC values.

- The ASL-Perfusion was the most significant MRI parameter in correlation with operative vascularity of meningiomas, with hyper vascular lesions showing nCBF ranging from 2.10 to 14.20 , intermediately vascular lesions ranging from 1.50 to 1.60 , and hypo vascular lesions ranging from 0.70 to 0.90 . ROC analysis showed 100\% sensitivity, specificity, PPV and NPV for $\mathrm{nCBF}>1.6$ in prediction of hyper vascular meningiomas, $\mathrm{nCBF} \leq 0.9$ for hypo vascular meningiomas with intermediately vascular lesions having $\mathrm{nCBF}$ between $>0.9$ and $\leq 1.6$ (Table 2 and Fig. 9).



(A)

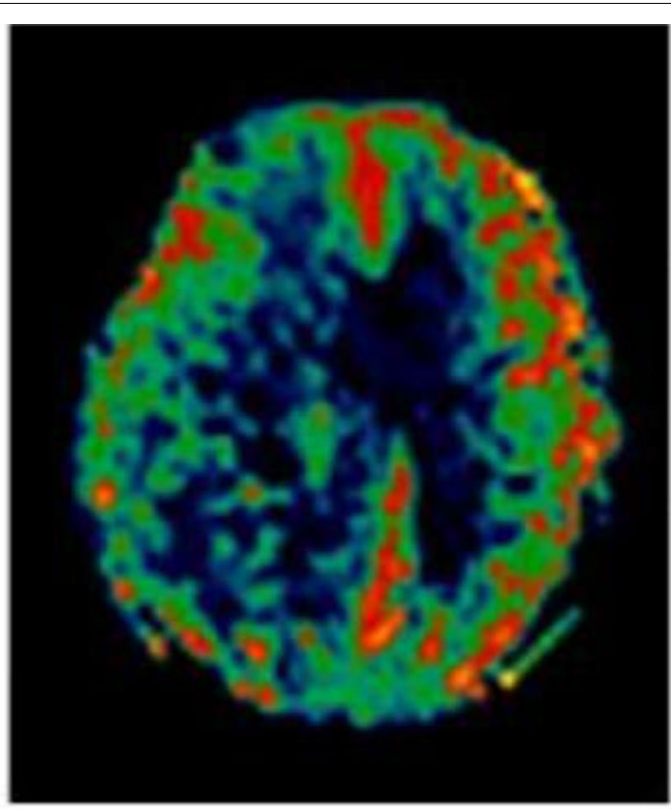

(B)

Fig. 8 MRl of hypovascular meningioma: A Axial T1 post contrast MRI showing intensely enhancing intraventricular meningioma. B Color coded ASL perfusion showing hypo perfusion of the lesion with $\mathrm{nCBF}$ of $0.7 \mathrm{ml} / 100 \mathrm{gm} / \mathrm{min}$, proved surgically to have low vascularity 
Table 2 Relation between meningioma operative vascularity and different MRI parameters

\begin{tabular}{|c|c|c|c|c|c|c|c|}
\hline \multirow[t]{3}{*}{ MRI parameters } & \multicolumn{6}{|c|}{ Meningioma Operative vascularity } & \multirow[t]{3}{*}{$p$} \\
\hline & \multicolumn{2}{|c|}{$\begin{array}{l}\text { Highly vascular } \\
(n=27)\end{array}$} & \multicolumn{2}{|c|}{$\begin{array}{l}\text { Intermediate vascularity } \\
(n=6)\end{array}$} & \multicolumn{2}{|c|}{$\begin{array}{l}\text { Hypo vascular } \\
(n=7)\end{array}$} & \\
\hline & No & $\%$ & No & $\%$ & No & $\%$ & \\
\hline \multicolumn{8}{|l|}{ T2 signal } \\
\hline Hyperintense & 18 & 66.7 & 1 & 16.7 & 2 & 28.6 & $p=0.003^{*}$ \\
\hline Isointense & 9 & 33.3 & 4 & 66.7 & 2 & 28.6 & \\
\hline Hypointense & 0 & 0.0 & 1 & 16.7 & 3 & 42.9 & \\
\hline Significance between groups & \multicolumn{6}{|c|}{$p_{1}=0.027^{*}, p_{2}=0.006^{*}, p_{3}=0.479$} & \\
\hline \multicolumn{8}{|l|}{ Diffusion } \\
\hline Free diffusion & 2 & 7.4 & 0 & 0.0 & 0 & 0.0 & \multirow[t]{3}{*}{$p=0.174$} \\
\hline Iso diffusion & 5 & 18.5 & 4 & 66.7 & 2 & 28.6 & \\
\hline Restricted & 20 & 74.1 & 2 & 33.3 & 5 & 71.4 & \\
\hline \multicolumn{8}{|l|}{$\operatorname{ADC}\left(\times 10^{-5} \mathrm{~cm}^{2} / \mathrm{s}\right)$} \\
\hline Min.-Max & \multicolumn{2}{|l|}{$0.60-1.30$} & \multicolumn{2}{|l|}{$0.70-1.20$} & \multicolumn{2}{|l|}{$0.70-1.20$} & \multirow[t]{3}{*}{$p=0.244$} \\
\hline Mean $\pm S D$ & $0.89 \pm 0.21$ & & $1.03 \pm 0.23$ & & $0.85 \pm 0.18$ & & \\
\hline Median & 0.85 & & 1.15 & & 0.80 & & \\
\hline \multicolumn{8}{|l|}{ ASL } \\
\hline Hyper perfusion & 27 & 100.0 & 0 & 0.0 & 0 & 0.0 & \multirow[t]{3}{*}{$p<0.001^{*}$} \\
\hline Iso perfusion & 0 & 0.0 & 6 & 100.0 & 0 & 0.0 & \\
\hline Hypo perfusion & 0 & 0.0 & 0 & 0.0 & 7 & 100.0 & \\
\hline Significance between groups & \multicolumn{6}{|c|}{$p_{1}<0.001^{*}, p_{2}<0.001^{*}, p_{3}=0.001^{*}$} & \\
\hline \multicolumn{8}{|l|}{$\mathrm{nCBF}$} \\
\hline Min.-Max & \multicolumn{2}{|l|}{$2.10-14.20$} & \multicolumn{2}{|l|}{$1.50-1.60$} & \multicolumn{2}{|l|}{$0.70-0.90$} & \multirow[t]{3}{*}{$p<0.001^{*}$} \\
\hline Mean $\pm S D$ & \multicolumn{2}{|l|}{$3.93 \pm 2.73$} & \multicolumn{2}{|l|}{$1.55 \pm 0.04$} & \multicolumn{2}{|l|}{$0.82 \pm 0.09$} & \\
\hline Median & 2.50 & & 1.55 & & 0.87 & & \\
\hline Significance between groups & \multicolumn{6}{|c|}{$p_{1}=0.002^{*}, p_{2}<0.001^{*}, p_{3}=0.313$} & \\
\hline
\end{tabular}

$\mathrm{ADC}$, apparent diffusion coefficient; $\mathrm{ASL}$, arterial spin labeling; $\mathrm{n}$, number of patients; $\mathrm{nCBF}$, normalized cerebral blood flow; $p$, $p$ value for association between different categories; $p_{1}, p$ value for association between hyper vascular and intermediately vascular; $p_{2}, p$ value for association between hyper vascular and hypo vascular; $p_{3}, p$ value for association between Intermediately vascular and hypo vascular meningioma; SD, standard deviation

*Statistically significant at $p \leq 0.05$

\section{Discussion}

Among central nervous system neoplasms, meningioma is one of the most common tumors, with surgical resection considered the curative therapy, however preoperative adequate assessment and planning is important in cases where meningioma present at difficult location for access and surgical resection [12].

Among neurosurgeons who are dealing with meningioma, its consistency is considered a critical concern, as soft tumors can be easily removed with suction and/ or a low intensity cavitron ultrasonic surgical aspirator (CUSA), with adequate debulking in easy quick surgery. On the other hand, firm meningiomas are usually difficult to be dissected from the adjacent critical structures that will affect the post-operative morbidity and mortality, so it is important for the surgeon to be aware about its consistency preoperatively for better selection of suitable equipment and proper access for better therapeutic outcome $[12,13]$.
Currently no single reproducible and objective method to predict meningioma consistency is available. Different imaging techniques and sequences were discussed in previous studies such as MR Elastography, MR spectroscopy, and fractional anisotropy with no clear consensus and till now there are conflicting results. Also, these recent techniques are usually expensive, not available everywhere in addition to complex interpretation [13].

In order to reach the aim of the current study we tried to answer the following questions: 1) Can conventional MRI detect meningioma consistency? and 2) Can MRI detect meningioma vascularity without angiography?

\section{Can conventional MRI detect meningioma consistency?}

In our study, 40 adult patients were analyzed for MRI characteristics and intraoperative tumor consistency. Although T2 signal showed significant correlation with consistency as most of hyperintense lesions were soft and 


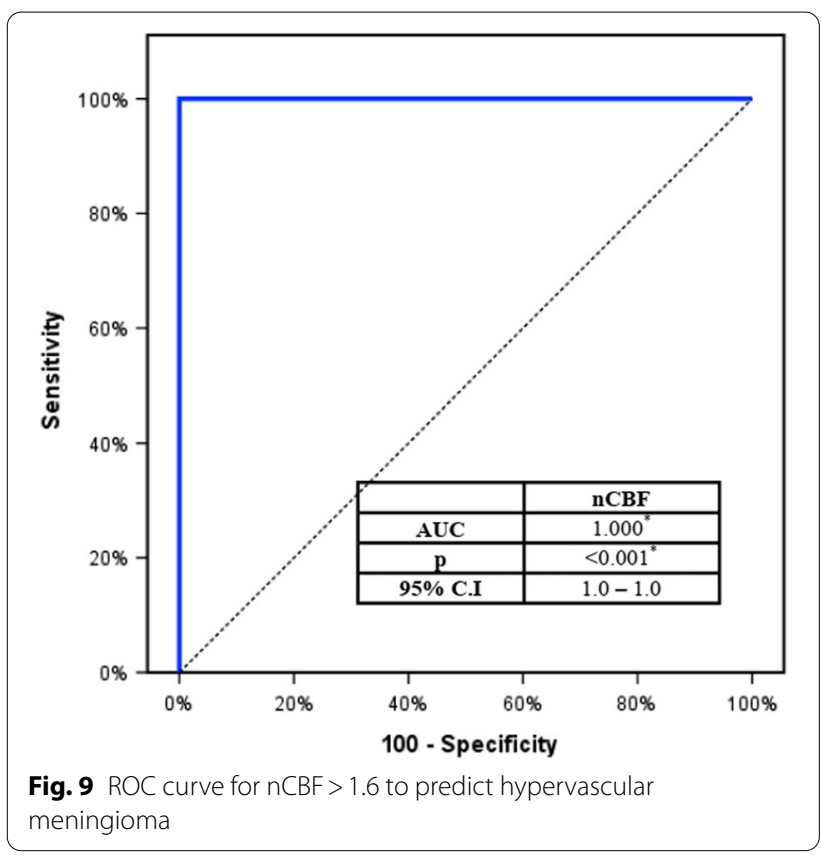

most of hypointense lesions were firm, yet still this was based on visual assessment that is completely subjective.

On the other hand, DWI with ADC which is more quantitative method gave some subjective data where restricted diffusion with mean ADC values $0.8 \times 10^{-5} \mathrm{~cm}^{2} / \mathrm{s}$ were detected in either soft or intermediate consistency meningiomas, yet no significant difference or cut off values was noted to differentiate soft from intermediate meningiomas.

Using routine conventional MRI, many studies were done on the correlation of MR signal intensity and meningioma consistency. The majority of these studies reached that no correlation between meningioma consistency and signal intensity in T1 or post contrast enhancement [7]. However, the T2WI showed the highest degree of correlation in different studies with results similar to the current one. Hoover et al. [14], and Sitthinamsuwan et al. [12], demonstrated a relationship between meningioma consistency and T2 signal intensity. In a relatively older study, Maiuri et al. [15], compared the T2 intensity of meningioma to cortex and found that $\mathrm{T} 2$ hyperintensity relative to cortex probably reflects soft consistency and syncytial or angioblastic subtypes.

Nevertheless, this correlation with imaging intensity has not been established. Kasoff et al. [16], and Kashimura et al. [17], concluded that there is no statistically significant correlation between consistency of meningioma and T2 intensity. They attributed this to the possibility of using different MRI machines, sequences, and protocols. Also, in all of these studies, signal intensity was not assessed quantitatively. Additionally, in most of these studies there was no specific definition of hypo or hyperintensity.

For better reproducibility, an objective method to predict tumor consistency preoperatively was defined using intraoperative tumor consistency grading. This initial idea was tested by Smith $\mathrm{K}$ et al. [11], in 20 patients as a pilot study to define values and investigate possible correlations. In the current study, we pursued validation of this method in a larger number of patients, using tumor/cerebellar peduncle T2-weighted imaging intensity (TCTI) ratios in correlation with intraoperative classification with suction and/or ultrasonic aspiration.

Intraoperative tumor consistency best correlated with TCTI ratios with $100 \%$ sensitivity, specificity, positive and negative predictive values. Soft tumors correlated with TCTI ratio $>1.6$ and were removed with suction alone or a CUSA amplitude $<40$. Soft tumors showed TCTI ranging from 1.75 to 2.87 with mean of 1.99 and median of 1.90. Firm tumors were correlated with TCTI ratios $\leq 1.2$ and necessitated a CUSA amplitude $>70$. Firm meningiomas showed TCTI ranging from 0.9 to 1.20 with mean of 1.09 and median of 1.10. Lastly intermediate consistency meningiomas best correlated with TCTI ratios $>1.2$ and $\leq 1$.6. Such intermediate consistency lesions showed TCTI ranging from 1.3 to 1.60 with mean of 1.50 and median of 1.53 .

These results matched with the previous two studies of Smith et al. [11, 13], the first of which was in 2015 on 20 meningioma patients where soft tumors correlated with TCTI ratio $>1.8$ with mean of 2.11 and median of 1.96. Firm tumors best correlated with TCTI ratios $\leq 1.0$ with mean of 0.6 and median of 0.62 . On the other hand, intermediate consistency meningiomas best correlated with TCTI ratios between 1.0 and 1.8 with mean of 1.51 and median of 1.6 [11].

Even more robust results were noted in the later study of Smith et al. [13], which was done in 2017 on 100 patients with intracranial meningiomas showing soft lesions to be correlated with TCTI ratio $>1.63$ with mean of 1.91 and median of 1.84. Firm tumors best correlated with TCTI ratios $\leq 1.27$ with mean of 1.01 and median of 0.88 . Lastly,intermediate consistency meningiomas correlated with TCTI ratios between 1.33 and 1.63 with mean of 1.49 and median of 1.5 [13].

The current study agrees with the previous two studies of Smith et al. [11, 13], regarding the objectivity of TCTI ratio which required no special equipment or interpretation. Such objectivity is likely to allow the general use of the TCTI ratios by radiologists to guide neurosurgeons complementing previous trials to assess the meningioma consistency quantitatively. 


\section{Can MRI detect meningioma vascularity without angiography?}

A subset of meningiomas exhibit hypervascular features, which increase their operative risk profile and can complicate operative planning. Excision of hypervascular symptomatic meningiomas may be preceded by preoperative embolization to reduce intraoperative bleeding, blood loss and facilitate surgical excision [18].

Historically, digital subtraction angiography (DSA) has been the standard method for identification of hypervascular meningiomas before operative intervention [19]. The radiologist typically depends on the presence of tumor blush on DSA; absence of this blush suggesting reduced or normal vascularity of the tumor [20]. Catheter angiography is generally considered safer than surgery and less invasive however, this technique is not without risk and inconvenience to the patient. Complications of DSA include risk of anesthesia, blood loss, groin hematoma, intracranial dissection, and ischemic stroke, in addition to exposure to radiation risk to the patient [21].

Perfusion imaging is a technique to assess the flow of blood at the tissue level and is widely used in imaging of brain tumors, mainly in the assessment of gliomas. Previous studies assessing meningiomas with dynamic susceptibility contrast MR perfusion detected a correlation between cerebral blood volume (CBV) and tumor vascularity histologically, including microvessel density and area $[22,23]$.

During the past decade, there has been growing interest in ASL-PWI, as it can quantify tissue perfusion without intravenous contrast injection, unlike the routine dynamic susceptibility contrast-enhanced PWI. The lack of IV contrast agents is considered beneficial particularly for patients with impaired renal function, in whom there is higher risk of developing nephrogenic systemic fibrosis after contrast administration [9].

The use of ASL-PWI in the assessment of brain tumors has been studied in different recent researches. For meningiomas, the relative CBF calculated from continuous ASL imaging was found to have a significant positive correlation with micro vessel area and thus to be easier to differentiate angiomatous subtype from others [24]. Similarly, in a recent study by Koizumi et al. [25], the absolute CBF from pseudo continuous ASL-PWI, rather than the relative CBF, was used to reflect the tumor vascularity. In their study, both the mean and maximum absolute CBF values were shown to have significant positive correlations with micro vascular density [25].

The current study tried to investigate whether ASL derived nCBF could serve as a reliable imaging biomarker for angiographic vascularity of meningiomas, in correlation with intraoperative tumor vascularity based on degree of intraoperative blood loss.

ASL showed significant correlation with operative vascularity of meningiomas, with hypervascular lesions showing $\mathrm{nCBF}$ ranging from 2.10 to $14.20 \mathrm{ml} / 100 \mathrm{gm} /$ $\mathrm{min}$, intermediately vascular lesions ranging from 1.50 to $1.60 \mathrm{ml} / 100 \mathrm{gm} / \mathrm{min}$, and hypo vascular lesions ranging from 0.70 to $0.90 \mathrm{ml} / 100 \mathrm{gm} / \mathrm{min}$. ROC analysis showed $100 \%$ sensitivity, specificity, PPV and NPV for $\mathrm{nCBF}>1.6$ in prediction of hyper vascular meningiomas, $\mathrm{nCBF} \leq 0.9$ for hypo vascular meningiomas with intermediately vascular lesions having $\mathrm{nCBF}$ between $>0.9$ and $\leq 1.6$.

The results of the current study were matching with the previous study of Yoo et al. [9], who studied ASL in 27 cases with meningioma in comparison with DSA to predict angiographic vascularity. In their study, angiographic vascularity was measured by DSA using a 4-point grading scale and meningiomas were divided into 2 groups: low vascularity (Grades 0 and $1 ; n=11$ ) and high vascularity (Grades 2 and 3; $n=16$ ). Authors concluded that, there is significant positive correlation between $\mathrm{nCBF}$ and angiographic vascularity $(\rho=0.718 ; p<0.001)$ where the high-vascularity group showed significantly higher $\mathrm{nCBF}$ than the low-vascularity group $(3.334 \pm 2.768$ and $0.909 \pm 0.468$, respectively; $p=0.003)$. At the optimal nCBF cutoff value of 1.73 , sensitivity and specificity for the differential diagnosis of the 2 groups were $69 \%$ and $100 \%$ respectively.

The authors of the same study also found that patients with high nCBF values from ASL maps would have high vascularity meningiomas and so they are considered as potential candidates for preoperative DSA and embolization. Given the high specificity $(100 \%)$ for differentiating between the high and low-vascularity groups at the optimal nCBF cutoff value, they concluded that the unnecessary DSA could have been avoided in the first place had the ASL findings been taken into consideration. Not only that, but the use of ASL-PWI as an initial screening tool is of benefit to reduce the length of hospital that will be reflected upon hospital resources. They concluded that ASL-PWI might predict angiographic vascularity, which will help to determine if there is need for preoperative embolization; thus, it may aid in selecting potential candidates for preoperative DSA and embolization.

\section{Points of strength}

All lesions in the current study had surgically and histologically proven diagnoses, and all MR imaging examinations were done at a high magnetic field of $3 \mathrm{~T}$ with higher signal to noise ratio (SNR) thus leading to decreased motion artifacts compared to $1.5 \mathrm{~T}$. The use of T2 sequence which required no special equipment or interpretation for detection of TCTI ratio allows 
generalization of the TCTI ratios to neuroradiologists and neurosurgeons. As there are differences in CBF values, so to use the quantitative measurement of $\mathrm{CBF}$ accurately, in the present study we measured a ratio of $\mathrm{CBF}$ values of white matter from the contralateral side as quantitative indicators to attain the nCBF.

\section{Limitations of the study}

Our study had some limitations. First, is small sample size, and validation of the optimal TCTI ratios and ASL derived $\mathrm{nCBF}$ cutoff values is needed in large case series. Second, no DSA nor dynamic susceptibility contrast perfusion studies were done for comparison with ASL in any of the included patients thus another objective imaging-based grading of meningiomas vascularity was missing. Third, in contrast to consistency, intraoperative assessment of meningioma vascularity was mainly based on degree on intraoperative blood loss, still with no definite grading system or scoring. Lastly, no correlation was done between MRI parameters of interest namely consistency and perfusion-based vascularity and the histopathological variants of meningiomas or their WHO grading; such correlation needs to be verified in future studies to identify the role of these MRI parameters in grading of meningiomas.

\section{Conclusions}

Prediction of meningioma consistency preoperatively is of importance for surgical planning and resection. TCTI ratio, showed a good correlation and, we hope, this will improve preoperative surgical planning for meningioma. Identification of vascularity of meningiomas preoperatively can significantly reduce intraoperative blood loss and facilitate surgical removal. ASL may provide a noninvasive, reliable means to predict angiographic vascularity of meningiomas and so it may be an alternative technique (for DSA and DSC-perfusion) to assess the perfusion of meningiomas, especially for patients when contrast agents are contraindicated.

\begin{abstract}
Abbreviations
TCTI: Tumor/cerebellar peduncle T2-weighted imaging intensity; ASL: Arterial spin labeling; ADC: Apparent diffusion coefficient; CUSA: Cavitron ultrasonic surgical aspirator; DWl: Diffusion weighted imaging; ECA: External carotid artery; GAD-DTPA: Gadolinium diethylene triamine pentacetic acid; NPV: Negative predictive value; nCBF: Normalized cerebral blood flow; PWI: Perfusion weighted imaging; PPV: Positive predictive value; PCASL: Pseudo continuous arterial spin labeling; ROC: Receiver operating characteristic curve; SWI: Susceptibility weighted imaging.
\end{abstract}

Acknowledgements

Not applicable.
Authors' contributions

$A B$ : editing the draft and revision of the final manuscript. AF: writing the clinical section. AA.: writing the draft DE: editing the draft. All authors have read and approved the final manuscript.

\section{Funding}

Not applicable.

\section{Availability of data and materials}

The datasets used and/or analyzed during the current study are available from the corresponding author on reasonable request.

\section{Declarations}

\section{Ethics approval and consent to participate}

Approval for this study was obtained from the Research Ethics Committee of Alexandria Faculty of Medicine (Ethics committee's reference number: 0305202, IRB No: 00012098, FWA No: 00018699). All study procedures were carried out in accordance with the Declaration of Helsinki regarding research involving human subjects. Written informed consent was obtained from the patients.

\section{Consent for publication}

Consent for the use of patients' data and medical record was obtained.

\section{Competing interests}

The authors declare that they have no competing interests.

\section{Author details}

${ }^{1}$ Department of Radiodiagnosis, Faculty of Medicine, University of Alexandria, Alexandria, Egypt. ${ }^{2}$ Department of Neurosurgery, Faculty of Medicine, University of Alexandria, Alexandria, Egypt.

Received: 26 August 2021 Accepted: 21 January 2022

Published online: 01 February 2022

\section{References}

1. Lin BJ, Chou KN, Kao HW (2014) Correlation between magnetic resonance imaging grading and pathological grading in meningioma. J Neuro surg 121(5):1201-1218

2. Zhang T, Jian-Min Y, Yong-Qi W, Dan-Dan Y, Long-Jiang F (2018) WHO grade I meningioma subtypes: MRI features and pathological analysis. Life Sci 15(213):50-56

3. Salah F, Tabbarah A, AlArab YN, Asmar K, Tamim H, Makki M et al (2019) Can CT and MRI features differentiate benign from malignant meningiomas? Clin Radiol 74(11):15-23

4. Zhao L, Zhao W, Hou Y, Wen C, Wang J, Wu P et al (2020) An overview of managements in meningiomas. Front Oncol 10:1523-1530

5. Goldbrunner R, Minniti G, Preusser M, Jenkinson M, Sallabanda K, Houdart E et al (2016) EANO guidelines for the diagnosis and treatment of Meningiomas. Lancet Oncol 17:383-391

6. Mark S, Steven Y, Benita T, D'Amore F, Alexander L, Kevin S et al (2016) Predicting meningioma consistency on preoperative neuroimaging studies. Neurosurg Clin N Am 27(2):145-154

7. Amy Y, Margaret P, Priti B, Raj K (2018) Can MRI predict meningioma consistency? A correlation with tumor pathology and systematic review. Neurosurg Rev 41(3):745-753

8. Toh CH, Wei KC, Chang CN, Peng YW, Ng SH, Wong HF et al (2014) Assessment of angiographic vascularity of meningiomas with dynamic susceptibility contrast-enhanced perfusion weighted imaging and diffusion tensor imaging. AJNR 35:263-269

9. Yoo R, Ji T, Young D, Jung H, Mi K, Hi S et al (2016) Utility of arterial spin labeling perfusion magnetic resonance imaging in prediction of angiographic vascularity of meningiomas. J Neurosurg 125(3):536-543

10. World Medical Association (2013) World Medical Association Declaration of Helsinki: ethical principles for medical research involving human subjects. JAMA 310(20):2191-2194 
11. Smith K, Leever J, Chamoun R (2015) Predicting consistency of meningioma by magnetic resonance imaging. J Neurol Surg B Skull Base 76(3):225-229

12. Sitthinamsuwan B, Inthira K, Sarun N, Prajak S, Wj T, Akkapong N (2012) Predictors of meningioma consistency: a study in 243 consecutive cases. Acta Neurochir 154(8):1383-1389

13. Smith KA, Leever JD, Hylton PD, Camarata PJ, Chamoun RB (2017) Meningioma consistency prediction utilizing tumor to cerebellar peduncle intensity on T2-weighted magnetic resonance imaging sequences: TCTI ratio. J Neurosurg 126(1):242-248

14. Hoover JM, Morris JM, Meyer FB (2011) Use of preoperative magnetic resonance imaging $\mathrm{T} 1$ and $\mathrm{T} 2$ sequences to determine intraoperative meningioma consistency. Surg Neurol Int 2:142

15. Maiuri F, laconetta G, de Divitiis O, Cirillo S, Di Salle F, De Caro ML (1999) Intracranial meningiomas: correlations between MR imaging and histology. Eur J Radiol 31:69-75

16. Kasoff SS, Spiller M, Valsamis MP, Lansen TA, Duffy KR, Koenig SH et al (1995) Relaxometry of noncalcified human meningiomas. Correlation with histology and solids content. Invest Radiol 30:49-55

17. Kashimura $\mathrm{H}$, Inoue $\mathrm{T}$, Ogasawara $\mathrm{K}$, Arai $\mathrm{H}$, Otawara $Y$, Kanbara $\mathrm{Y}$ et al (2007) Prediction of meningioma consistency using fractional anisotropy value measured by magnetic resonance imaging. J Neurosurg 107:784-787

18. Hiroshi M, Katsumi S, Junya T, Tadao S, Wataru S, Tetsuya Y (2018) Safety and efficacy of preoperative embolization in patients with meningioma. $J$ Neurol Surg B Skull Base 79(Suppl 4):328-333

19. James RF, Kramer DR, Page PS, Gaughen JR, Martin LB, Mack WJ (2016) Strategic and technical considerations for the endovascular embolization of intracranial meningiomas. Neurosurg Clin N Am 27:155-166

20. Ding D, Raper DM, Starke RM (2015) Preoperative embolization for intracranial meningiomas: selecting optimal targets based on tumor anatomy and angioarchitecture. Clin Neuroradiol 25:327-328

21. Vera M, Mingming M, Samantha H, Jeremy H, Michael I (2019) Arterial spin-labeling MRI identifies hyper vascular meningioma. AJR 213(5):1124-1128

22. Kimura $\mathrm{H}$, Takeuchi $\mathrm{H}$, Koshimoto $\mathrm{Y}$, Arishima $\mathrm{H}$, Uematsu $\mathrm{H}$, Kawamura $Y$ et al (2006) Perfusion imaging of meningioma by using continuous arterial spin-labeling: comparison with dynamic susceptibility-weighted contrast-enhanced MR images and histopathologic features. AJNR 27(1):85-93

23. Shi R, Jiang T, Si L, Li M (2016) Correlations of magnetic resonance, perfusion-weighed imaging parameters and microvessel density in meningioma. J BUON 21(3):709-713

24. Xin Q, Hyun Grace K, Danny JW, Noriko S, Michael L, Ali S et al (2017) Application of arterial spin labeling perfusion MRI to differentiate benign from malignant intracranial meningiomas. Eur J Radiol 97:31-36

25. Koizumi S, Sakai N, Kawaji H, Takehara Y, Yamashita S, Sakahara H et al (2015) Pseudo-continuous arterial spin labeling reflects vascular density and differentiates angiomatous meningiomas from non-angiomatous meningiomas. J Neurooncol 121:549-556

\section{Publisher's Note}

Springer Nature remains neutral with regard to jurisdictional claims in published maps and institutional affiliations.

\section{Submit your manuscript to a SpringerOpen ${ }^{\odot}$ journal and benefit from:}

- Convenient online submission

- Rigorous peer review

- Open access: articles freely available online

- High visibility within the field

- Retaining the copyright to your article 